\title{
Fourth-Order Pattern Forming PDEs: Partial and Approximate Symmetries
}

\author{
Sameerah Jamal ${ }^{a}$ and Andrew G. Johnpillai ${ }^{b}$ \\ ${ }^{a}$ School of Mathematics, University of the Witwatersrand, \\ Johannesburg, South Africa \\ ${ }^{b}$ Department of Mathematics, Eastern University, \\ 30350, Sri Lanka \\ E-mail(corresp.): Sameerah.Jamal@wits.ac.za \\ E-mail: andrewgratienj@yahoo.com
}

Received April 18, 2019; revised January 11, 2020; accepted January 12, 2020

\begin{abstract}
This paper considers pattern forming nonlinear models arising in the study of thermal convection and continuous media. A primary method for the derivation of symmetries and conservation laws is Noether's theorem. However, in the absence of a Lagrangian for the equations investigated, we propose the use of partial Lagrangians within the framework of calculating conservation laws. Additionally, a nonlinear Kuramoto-Sivashinsky equation is recast into an equation possessing a perturbation term. To achieve this, the knowledge of approximate transformations on the admissible coefficient parameters is required. A perturbation parameter is suitably chosen to allow for the construction of nontrivial approximate symmetries. It is demonstrated that this selection provides approximate solutions.
\end{abstract}

Keywords: pattern formation, optimal system of one-dimensional subalgebras, Lie symmetries, exact solutions.

AMS Subject Classification: 35C05; 35C06; 76M60; 35A09.

\section{Introduction}

The family of nonlinear fourth-order partial differential equations (PDEs)

$$
u_{t}+\lambda u u_{x}+\alpha u_{x x}+\beta u_{x x x}+\gamma u_{x x x x}+\zeta(u)=0, \quad \alpha, \beta, \gamma, \lambda-\text { constants, }
$$

describes several fundamental phenomena in physical processes. Indeed, it encompasses the generalized Korteweg-de Vries equation $(\alpha=\zeta=\gamma=0, \beta=$ $\lambda=1)$, Burger's equation $(\beta=\zeta=\gamma=0)$, Benny's equation [3] $(\alpha=\lambda=$ 
$\gamma=1, \zeta=0)$ to describe instabilities on falling fluid films, among many others. Broadly speaking, over a wide range of parameter values, this family of equations possesses solutions that take the form of patterns such as pulses [15] and spatio-temporal pattern formation in extended systems.

Our interest lies in a subclass of these equations called the Swift-Hohenberg (SH) [28] and Kuramoto-Sivashinsky (KS) equations [17,27] that exhibit interesting pattern formations. The former was introduced as a simple model for the Rayleigh-Bénard instability of roll waves, but has since appeared in connection with Taylor-Couette flow [25] and in the study of lasers [18]. It is considered a model equation for various higher-order parabolic models arising in many contexts, such as the phase field models [4] and the extended Fisher-Kolmogorov equation [6].

The latter PDE, the KS equation is one of the simplest physically interesting nonlinear systems and is frequently encountered in the study of continuous media which exhibits a chaotic behavior. In the literature, it has offered insight on the turbulence in full-fledged Navier-Stokes boundary shear flows [7], descriptions of stability of flame fronts, reaction diffusion systems, long waves on the interface between two viscous fluids and unstable drift waves in plasmas.

These equations stem from different applications, but share the property unique to only a few physical mechanisms: simple evolutionary PDEs that produce intricate patterns. In this paper, we will perform a symmetry and conservation law analysis with the aim of exploring the pattern mechanisms via group-invariant solutions and visual representations.

In the past, solutions of these and related equations have been found using numerical methods, see $[5,16]$ and references therein. Recent studies involving conservation laws and symmetries have provided interesting results for stream functions [10], beams [13], biological models [21], nonlinear systems [2] and diffusion equations [11].

We employ the use of partial Lagrangians in our analysis. Despite a critic [26] of the method, it has proven to be quite useful and has an extended application to Hamiltonians [20]. The method not only gives rise to conserved quantities, but may also be used to obtain new closed form solutions [19]. Several other works in the literature have involved partial Lagrangians, namely [3,8,12].

The plan of the paper is as follows. In Section 2, the partial Noether operators of the $\mathrm{SH}$ equation are discovered. Section 3 discusses various exact solutions of the KS class of equations based on classical and approximate symmetries along with a conservation law. The conclusion and references are presented subsequently.

\section{The Swift-Hohenberg equation}

Explicitly, the parabolic Swift-Hohenberg equation

$$
u_{t}=(-1+k) u-f(u)-2 u_{x x}-u_{x x x x}, \quad k-\text { constant },
$$

is a model pattern-forming equation for a fluid that is thermally convectional. The bifurcation parameter $k$ plays the role of a temperature knob, measuring how far the temperature is above the minimum temperature difference required 
for convection. Therefore, for $k<0$ the heating at the bottom of the fluid is too small to cause convection, while for $k>0$ convection occurs.

The Lie point symmetries of this equation are $X_{1}=\partial_{x}, X_{2}=\partial_{t}$. Equation (2.1) does not admit any conservation law multipliers and does not possess a variational principle. Hence, in order to obtain conservation laws, one may resort to other methods. In particular search for partial Noether operators [14].

\subsection{Partial Noether symmetries and conservation laws}

Equation (2.1) is an evolution equation associated with the partial Lagrangian

$$
L=\frac{1}{2} u_{x x}^{2}-u_{x}^{2}+\int f(u) d u+\frac{1}{2}(1-k) u^{2}
$$

with a corresponding partial Noether operator

$$
\bar{X}=\xi^{1}(x, t, u) \partial_{t}+\xi^{2}(x, t, u) \partial_{x}+\eta(x, t, u) \partial_{u} .
$$

The coefficient functions $\xi^{1}, \xi^{2}$ and $\eta$ are calculated by solving the partial Noether determining equation

$$
\bar{X} L+L D_{i} \xi^{i}=W \frac{\delta L}{\delta u}+D_{i} B^{i}
$$

where $W$ is the characteristic of $\bar{X}, B^{i}=B^{i}(x, t, u)$ and $\frac{\delta}{\delta u}$ is the EulerLagrange operator. Since $\xi^{1}, \xi^{2}$ and $\eta$ are independent of the derivatives of $u$, the coefficients of like derivatives of $u$ in the partial Noether determining equation can be equated to yield an over determined system of linear PDEs. Therefore, the determining system for the partial Noether operators yield

$$
\begin{aligned}
& \xi_{u}^{1}=0, \xi_{x}^{1}=0, \xi_{u}^{2}=0, \xi^{1}=0, \eta_{u u}=0, \\
& 2 \eta_{x u}-\xi_{x x}^{2}=0, \eta_{u}-\frac{3}{2} \xi_{x}^{2}=0, \eta_{x x}=0, \\
& \xi^{2}=0, \eta_{u}=0,-\eta+B_{u}^{1}=0,2 \eta_{x}+B_{u}^{2}=0, \\
& \eta f(u)+(1-k) \eta u=B_{t}^{1}+B_{x}^{2} .
\end{aligned}
$$

The analysis of the determining equation gives us partial Noether operators and gauge terms $\left(B^{1}, B^{2}\right)$ for $f(u)=f_{0} u+f_{1}$, where $f_{0}, f_{1}$ are constants. That is:

$$
\begin{aligned}
\bar{X}_{1}= & x e^{\left[f_{0}+(1-k)\right] t} \frac{\partial}{\partial u}, \quad B^{1}=\left[u+\frac{f_{1}}{f_{0}+(1-k)}\right] \\
& \times x e^{\left[f_{0}+(1-k)\right] t}+F x-t, \quad B^{2}=-2 u e^{\left[f_{0}+(1-k)\right] t}+x . \\
\bar{X}_{2}= & e^{\left[f_{0}+(1-k)\right] t} \frac{\partial}{\partial u}, \quad B^{1}=\left[u+\frac{f_{1}}{f_{0}+(1-k)}\right] \\
& \times e^{\left[f_{0}+(1-k)\right] t}+F x-t, \quad B^{2}=x .
\end{aligned}
$$

The respective conserved vectors corresponding to the partial Noether operators and the gauge terms in (2.2) and (2.3) are found to be

$$
T^{1}=\left[u+\frac{f_{1}}{f_{0}+(1-k)}\right] x e^{\left[f_{0}+(1-k)\right] t}+F x-t, \quad T^{2}=-2 u e^{\left[f_{0}+(1-k)\right] t}
$$




$$
\begin{aligned}
& +x+2 x e^{\left[f_{0}+(1-k)\right] t} u_{x}-e^{\left[f_{0}+(1-k)\right] t} u_{x x}+x e^{\left[f_{0}+(1-k)\right] t} u_{x x x}, \\
T^{1}= & {\left[u+\frac{f_{1}}{f_{0}+(1-k)}\right] e^{\left[f_{0}+(1-k)\right] t}+F x-t, } \\
T^{2}= & x+2 e^{\left[f_{0}+(1-k)\right] t} u_{x}+e^{\left[f_{0}+(1-k)\right] t} u_{x x x} .
\end{aligned}
$$

\section{The Kuramoto-Sivashinsky equations}

Consider the following class of pattern forming equations

$$
u_{t}+u u_{x}+\alpha u_{x x}+\beta u_{x x x}+\gamma u_{x x x x}+\sigma u=0, \quad \sigma-\text { constant. }
$$

This pattern forming PDE (commonly called the generalized KuramotoSivashinsky (gKS) equation) admits a conservation law multiplier (analogous to an integrating factor) $Q=\mathrm{e}^{\sigma t}$. Hence, it is straightforward to construct the conserved density and flux, viz.

$$
T^{t}=-\mathrm{e}^{\sigma t} u, T^{x}=-\frac{1}{2} \mathrm{e}^{\sigma t}\left(u^{2}+2 \alpha u_{x}+2 \beta u_{x x}+2 \gamma u_{x x x}\right) .
$$

Next, we consider special parameter values that enlarge the symmetry algebra. The classifications of subgroups of Lie point symmetry groups is an essential part in the analysis of differential equations. A classification of all three- and four- dimensional subalgebras can be found in [24]. Classifications facilitate an efficient computation of group-invariant solutions, while removing the occurrence of equivalent solutions. To this end, the classifications of subgroups of symmetries is performed with the use of adjoint representations.

\subsection{Case I: $\alpha=\beta=\sigma=0$.}

The parameters of this case provide the generalized Burgers-KdV equation. Recently in [23], a group analysis focused on variable-coefficient Burgers-KdV equations was performed. This study included the generalized Burgers-KdV equation (with constant coefficient), but did not provide the solutions, and so to the best of our knowledge, the results below have not appeared elsewhere. This case admits the symmetries

$$
X_{1}, X_{2}, X_{3}=t \partial_{x}+\partial_{u}, X_{4}=4 t \partial_{t}-3 u \partial_{u}+x \partial_{x} .
$$

We also point out that other symmetry classifications involving the parameters $\alpha, \beta$ have either appeared in numerous places in the literature (for one such example, see the discussion and references contained in [23]) or produce no further results of interest. The parameter $\sigma$ can be considered as a linear damping term.

For this case, we present the optimal system of one-dimensional subalgebras using the results in Table 1 . The method used here for obtaining the one-dimensional optimal system of subalgebras is that described in [22]. This approach, in essence, is taking a general element from the Lie algebra and reducing it to its simplest equivalent form by applying carefully chosen adjoint 
transformations

$$
\operatorname{Ad}\left(\exp \left(\varepsilon X_{i}\right)\right) X_{j}=X_{j}-\varepsilon\left[X_{i}, X_{j}\right]+\frac{1}{2} \varepsilon^{2}\left[X_{i},\left[X_{i}, X_{j}\right]\right]-\cdots .
$$

In order to limit the volume of the results, we omit the lengthy details of the calculations and simply list the one-dimensional optimal system of subalgebras which comprise of the vector fields: $\left\{X_{1}\right\},\left\{X_{2}\right\},\left\{X_{3}+\varepsilon X_{2}\right\},\left\{X_{4}\right\}$, where $\varepsilon=0, \pm 1$. As mentioned above, inequivalent group-invariant solutions are obtainable using one-dimensional optimal systems - we present the complete symmetry reductions below.

Table 1. Lie brackets and adjoints of the vector fields.

\begin{tabular}{ccccc}
\hline$\left[X_{i}, X_{j}\right]$ & $X_{1}$ & $X_{2}$ & $X_{3}$ & $X_{4}$ \\
\hline$X_{1}$ & 0 & 0 & 0 & $X_{1}$ \\
$X_{2}$ & 0 & 0 & $X_{1}$ & $4 X_{2}$ \\
$X_{3}$ & 0 & $-X_{1}$ & 0 & $-3 X_{3}$ \\
$X_{4}$ & $-X_{1}$ & $-4 X_{2}$ & $3 X_{3}$ & 0 \\
& & & & $X_{4}$ \\
\hline$A d\left(\varepsilon X_{i}, X_{j}\right)$ & $X_{1}$ & $X_{2}$ & $X_{3}$ & $-\varepsilon X_{1}+X_{4}$ \\
$X_{1}$ & $X_{1}$ & $X_{2}$ & $X_{3}$ & $-4 \varepsilon X_{2}+X_{4}$ \\
$X_{2}$ & $X_{1}$ & $X_{2}$ & $-\varepsilon X_{1}+X_{3}$ & $3 \varepsilon X_{3}+X_{4}$ \\
$X_{3}$ & $X_{1}$ & $\varepsilon X_{1}+X_{2}$ & $X_{3}$ & $X_{4}$ \\
$X_{4}$ & $e^{\varepsilon} X_{1}$ & $e^{4 \varepsilon} X_{2}$ & $e^{-3 \varepsilon} X_{3}$ & \\
\hline
\end{tabular}

- The symmetry $X_{1}$ leads to the solution $u(x, t)=h(t)$, where $h^{\prime}(t)=0$, or simply $h(t)=A_{1}=$ const.

- The symmetry $X_{2}$ leads to the solution $u(x, t)=f(x)$ and the reduced equation

$$
f(x) f^{\prime}(x)+\gamma f^{\prime \prime \prime \prime}(x)=0 .
$$

In turn, Equation (3.2) possesses the symmetries

$$
X_{1}^{*}=\partial_{x}, X_{2}^{*}=f \partial_{f}-\frac{1}{3} x \partial_{x},
$$

so a reduction using $X_{2}^{*}$ leads to the solution $f(x)=120 \gamma / x^{3}$.

- $X_{3}+\varepsilon X_{2}$ leads to the similarity variables

$$
u(x, t)=\frac{t+\varepsilon g(\varrho)}{\epsilon}, \quad \varrho=\frac{-t^{2}+2 x \varepsilon}{2 \varepsilon},
$$

where $g(\varrho)$ satisfies the equation

$$
1+\varepsilon g(\varrho) g^{\prime}(\varrho)+\gamma \varepsilon g^{\prime \prime \prime \prime}(\varrho)=0 .
$$

- $X_{4}$ admits the solution $u(x, t)=\frac{j(\psi)}{t^{3 / 4}}, \quad \psi=\frac{x}{t^{1 / 4}}$. In this case the reduced equation is

$$
j(\psi)\left(3-4 j^{\prime}(\psi)\right)+\psi j^{\prime}(\psi)-4 \gamma j^{\prime \prime \prime \prime}(\psi)=0 .
$$




\subsection{Case II: $\alpha, \beta, \sigma, \gamma \neq 0$.}

Here, the Lie point symmetries are

$$
X_{1}, X_{2}, X_{5}=\mathrm{e}^{-\sigma t}\left(\sigma \partial_{u}-\partial_{x}\right) .
$$

Proceeding in the same manner as the previous subsection but with the use of Table 2, the optimal system of one-dimensional subalgebras are $\left\{X_{2}+\right.$ $\left.a X_{1}\right\},\left\{X_{5}+\varepsilon X_{1}\right\},\left\{X_{1}\right\}$, where $a$ is an arbitrary constant and $\varepsilon=0, \pm 1$.

Table 2. Lie brackets and adjoints of the vector fields.

\begin{tabular}{cccc}
\hline$\left[X_{i}, X_{j}\right]$ & $X_{1}$ & $X_{2}$ & $X_{5}$ \\
\hline$X_{1}$ & 0 & 0 & 0 \\
$X_{2}$ & 0 & 0 & $-\sigma X_{5}$ \\
$X_{5}$ & 0 & $\sigma X_{5}$ & 0 \\
& & & \\
\hline$A d\left(\varepsilon X_{i}, X_{j}\right)$ & $X_{1}$ & $X_{2}$ & $X_{5}$ \\
\hline$X_{1}$ & $X_{1}$ & $X_{2}$ & $X_{5}$ \\
$X_{2}$ & $X_{1}$ & $X_{2}$ & $e^{\varepsilon \sigma} X_{5}$ \\
$X_{5}$ & $X_{1}$ & $X_{2}-\varepsilon \sigma X_{5}$ & $X_{5}$ \\
\hline
\end{tabular}

The corresponding group-invariant solutions are as follows.

- $X_{2}+a X_{1}$ provides the travelling wave solution, with the invariants

$$
\rho=x-a t, \quad u(x, t)=w(\rho) .
$$

The gKS reduces to $-a w^{\prime}+w\left(\sigma+w^{\prime}\right)+\alpha w^{\prime \prime}+\beta w^{\prime \prime \prime}+\gamma w^{\prime \prime \prime \prime}=0$.

- $X_{5}+\varepsilon X_{1}$ leads to the similarity variable

$$
u(x, t)=\frac{x \sigma}{-1+e^{t \sigma \varepsilon}}+n(t),
$$

and the reduction

$$
e^{t \sigma} \varepsilon \sigma n(t)+\left(-1+e^{t \sigma} \varepsilon\right) n^{\prime}(t)=0,
$$

that has the solution

$$
n(t)=\frac{A_{2}}{1-e^{t \sigma} \varepsilon}, \quad A_{2}-\text { constant. }
$$

Figure 1 showcase various density plots of the solution (3.3) in order to capture the distribution or pattern of the data values.

- Reduction by $X_{1}$ yields

$$
u(x, t)=m(t),
$$

where $\sigma m(t)+m^{\prime}(t)=0$. The solution of this ODE is

$$
m(t)=A_{3} e^{-t \sigma}, \quad A_{3}-\text { constant. }
$$




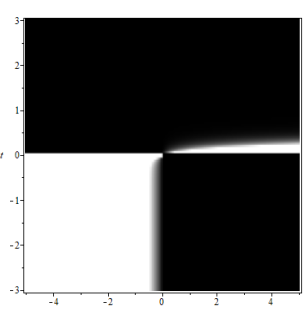

a)

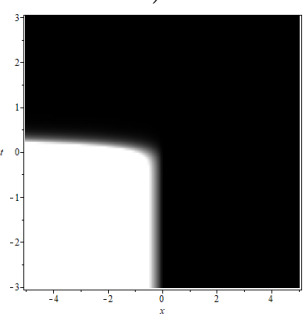

c)

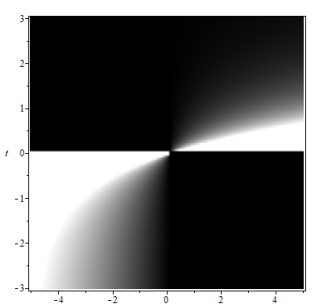

b)

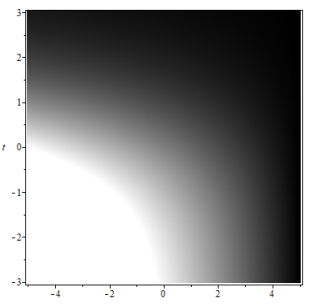

d)

Figure 1. Analytical solutions of (3.3) are presented, we select the parameter values: a) $A_{2}=0.01, \sigma=10, \varepsilon=+1$, b) $A_{2}=0.1, \sigma=1, \varepsilon=+1$, c) $A_{2}=0.01, \sigma=10, \varepsilon=-1$, d) $A_{2}=5, \sigma=1, \varepsilon=-1$.

\subsubsection{Approximate solutions}

Suppose we let $\beta=0$ and $\alpha=\gamma=1$. If we treat $\sigma \ll 1$ as a small parameter, then we obtain approximate symmetries $X=X^{0}+\sigma X^{1}+O\left(\sigma^{2}\right)$, where

$$
X^{A}=\xi_{t}^{A}(t, x, u) \partial_{t}+\xi_{x}^{A}(t, x, u) \partial_{x}+\eta^{A}(t, x, u) \partial_{u}, A=0,1 .
$$

The $X^{0}$ are determined in the usual way but with $\sigma=0$. The $X^{1}$ are calculated by solving the determining equation for deformations

$$
\left.X^{1}\left(u_{t}+u u_{x}+u_{x x}+u_{x x x x}\right)\right|_{u_{t}+u u_{x}+u_{x x}+u_{x x x x}=0}+H=0,
$$

where

$$
H=\frac{1}{\sigma} X^{0}\left(u_{t}+u u_{x}+u_{x x}+u_{x x x x}+\sigma u\right) \text { modulo Equation(3.1). }
$$

We find $H=H_{0}$ a constant and the determining system (3.4) is

$$
\begin{gathered}
\xi_{x, u}^{1}=0, \xi_{t, u}^{1}=0, \xi_{t, x}^{1}=0, \eta_{, u u}^{1}=0, \\
\xi_{t, t}^{1}-4 \xi_{x, x}^{1}=0, \xi_{t, t}^{1}-2 \xi_{x, x}^{1}-4 \xi_{x, x x x}^{1}+6 \eta_{, u x x}^{1}=0, \\
2 \eta_{, u x}^{1}-3 \xi_{x, x x}^{1}=0, u \eta_{, x}^{1}+\eta_{, t}^{1}+\eta_{, x x}^{1}+\eta_{, x x x x}^{1}+H_{0}=0, \\
u \xi_{t, t}^{1}+\eta^{1}-\xi_{x, t}^{1}-\xi_{x, x x}^{1}-\xi_{x, x x x x}^{1}+2 \eta_{, u x}^{1}+4 \eta_{, u x x x}^{1}-\xi_{x, x}^{1}=0 .
\end{gathered}
$$

The solution of the above system provides the approximate symmetries

$$
X_{1}, X_{2}, X_{6}=\sigma\left(\partial_{u}+t \partial_{x}\right), X_{7}=(-\sigma t+1) \partial_{u}+\left(t-\frac{\sigma}{2} t^{2}\right) \partial_{x}
$$


Now, approximate symmetries lead to approximate reductions and solutions $[1,9]$. The approximate solutions of Equation (3.1) are presented below.

- The reduction by $X_{7}$ results in the change of variables

$$
t=\bar{t}, \quad u(x, t)=v-\left(\frac{2 x-2 t x \sigma}{\sigma \bar{t}^{2}-2 \bar{t}}\right) .
$$

Equation (3.1) reduces to

$$
\bar{t}^{2} v(\bar{t}) \sigma^{2}+\bar{t}^{2} v_{\bar{t}} \sigma-2 v_{\bar{t}} \bar{t}-2 v=0,
$$

that solves to give

$$
v=\frac{c_{0} \mathrm{e}^{-\sigma \bar{t}}}{\bar{t}(\sigma \bar{t}-2)}, \quad c_{0}-\text { constant. }
$$

- From the reduction by $c_{1} X_{1}+c_{2} X_{6}$ ( $c_{1}, c_{2}$ - constants), we find

$$
t=\bar{t}, \quad u(x, t)=w+\frac{c_{2} \sigma x}{\left(c_{2} \sigma \bar{t}+c_{1}\right)} .
$$

In this case, Equation (3.1) reduces to

$$
c_{2} \bar{t} w \sigma^{2}+c_{2} \bar{t} w_{\bar{t}} \sigma+w c_{2} \sigma+c_{1} w \sigma+w_{t} c_{1}=0,
$$

that gives $w=c_{4} \mathrm{e}^{-\sigma \bar{t}} /\left(c_{2} \bar{t} \sigma+c_{1}\right), \quad c_{4}-$ constant.

Figure 2 provides data visualization of the solution (3.5).

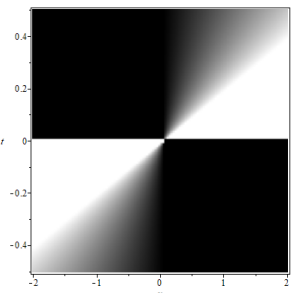

a)

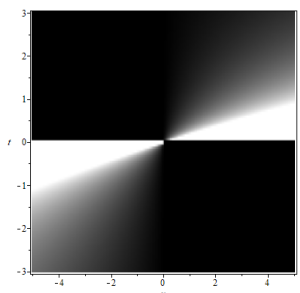

b)

Figure 2. Analytical solutions of (3.5) are depicted, we select the parameter values: a) $\left.c_{0}=0.1, \sigma=0.0001, \mathrm{~b}\right) c_{0}=0.01, \sigma=0.2$.

\section{Conclusions}

A wide range of symmetry methods are applied to several renowned differential equations arising in pattern formations. Lie point symmetries of the Swift-Hohenberg and a class of Kuramoto-Sivashinsky models are computed. One-dimensional inequivalent subalgebras of the respective maximal Lie invariance algebras are classified. Based on our classification, we determine groupinvariant solutions of the investigated differential equations. Since one of the 
models considered lacked a variational principle we showed that a conservation law is obtainable via partial Lagrangians. Secondly, we selected a perturbation parameter for the KS model. This lead to the derivation of nontrivial approximate point symmetries and solutions.

\section{Acknowledgements}

SJ acknowledges the financial support of the National Research Foundation of South Africa (99279).

\section{References}

[1] V.A. Baikov, R.K. Gazizov and N.H. Ibragimov (Eds). CRC Handbook of Lie Group Analysis of Differential Equations. Vol. 3. CRC Press, Boca Raton, Florida, 1996.

[2] J. Belmonte-Beitia, V.M. Pérez-García, V. Vekslerchik and P.J. Torres. Lie symmetries and solitons in nonlinear systems with spatially inhomogeneous nonlinearities. Phys. Rev. Lett., 98(064102), 2007. https://doi.org/10.1103/PhysRevLett.98.064102.

[3] D.J. Benney. Long waves on liquid films. J. Math. and Phys., 45:150-155, 1966. https://doi.org/10.1002/sapm1966451150.

[4] G. Caginal and P.C. Fife. Higher order phase field models and detailed anisotropy. Phys. Rev. B, 34:4940-4943, 1986. https://doi.org/10.1103/PhysRevB.34.4940.

[5] P. Collet and J.P. Eckmann. Instabilities and fronts in extended systems. Princeton Series in Physics, Princeton University Press, New Jersey, 1990. https://doi.org/10.1515/9781400861026.

[6] G.T. Dee and W. van Saarloos. Bistable systems with propagating fronts leading to pattern formation. Phys. Rev. Lett., 60:2641-2644, 1988. https://doi.org/10.1103/PhysRevLett.60.2641.

[7] P. Holmes, J.L. Lumley and G. Berkooz. Turbulence, Coherent Structures, Dynamical Systems and Symmetry. Cambridge Univ. Press, Cambridge, 1996. https://doi.org/10.1017/CBO9780511622700.

[8] I. Hussain, F.M. Mahomed and A. Qadir. Approximate partial Noether operators of the Schwarzschild spacetime. J. Non. Math. Phys., 17(1):13-25, 2013. https://doi.org/10.1142/S1402925110000556.

[9] N.H. Ibragimov and V.F. Kovalev. Approximate and Renormgroup Symmetries. Springer-Verlag, Berlin, 2009. https://doi.org/10.1007/978-3-642-00228-1.

[10] S. Jamal. Solutions of quasi-geostrophic turbulence in multilayered configurations. Quaest. Math., 41:409-421, 2018. https://doi.org/10.2989/16073606.2017.1383947.

[11] S. Jamal and A. Mathebula. Generalized symmetries and recursive operators of some diffusive equations. Bull. Malays. Math. Sci. Soc., 42:697-706, 2019. https://doi.org/10.1007/s40840-017-0510-z.

[12] S.U. Jing-Rui, Z. Shun-Li and L. Ji-Na. Approximate Noether-type symmetries and conservation laws via partial Lagrangians for nonlinear wave equation with damping. Comm. Theor. Phys., 53:37-42, 2010. https://doi.org/10.1088/02536102/53/1/08. 
[13] A.G. Johnpillai, K.S. Mahomed, C. Harley and F.M. Mahomed. Noether symmetry analysis of the dynamic Euler-Bernoulli beam equation. Z. Naturforsch, 71(5):447-456, 2016. https://doi.org/10.1515/zna-2015-0292.

[14] A.H. Kara and F.M. Mahomed. Noether-type symmetries and conservation laws via partial Lagrangians. Non. Dyn., 45:367-383, 2006. https://doi.org/10.1007/s11071-005-9013-9.

[15] T. Kawahara and S. Toh. Pulse interactions in an unstable dissipativedispersive nonlinear system. Phys. Fluids, 31:2103-2111, 1987. https://doi.org/10.1063/1.866610.

[16] A.H. Khater and R.S. Temsah. Numerical solutions of the generalized KuramotoSivashinsky equation by Chebyshev spectral collocation methods. Comp. Math. Appl., 56:1465-1472, 2008. https://doi.org/10.1016/j.camwa.2008.03.013.

[17] Y. Kuramoto and T. Tsuzuki. Persistent propagation of concentration waves in dissipative media far from thermal equilibrium. Prog. Theor. Phys., 55:356, 1976. https://doi.org/10.1143/PTP.55.356.

[18] J. Lega, J.V. Moloney and A.C. Newell. Swift-Hohenberg equation for lasers. Phys. Rev. Lett., 73:2978-2981, 1994. https://doi.org/10.1103/PhysRevLett.73.2978.

[19] R. Naz. The applications of the partial Hamiltonian approach to mechanics and other areas. Int. J. Non. Mech., 86:1-6, 2016. https://doi.org/10.1016/j.ijnonlinmec.2016.07.009.

[20] R. Naz, F.M. Mahomed and A. Chaudhry. A partial Hamiltonian approach for current value Hamiltonian systems. Comm. Non. Sci. Num. Sim., 19(10):36003610, 2014. https://doi.org/10.1016/j.cnsns.2014.03.023.

[21] M.C Nucci and G. Sanchini. Noether symmetries quantization and superintegrability of biological models. Symmetry, 8:1-9, 2016. https://doi.org/10.3390/sym8120155.

[22] P. Olver. Application of Lie Groups to Differential Equations. Springer, New York, 1993. https://doi.org/10.1007/978-1-4612-4350-2.

[23] S. Opanasenko, A. Bihlo and R.O. Popovych. Group analysis of general Burgers-Korteweg-de Vries equations. J. Math. Phys., 58:081511, 2017. https://doi.org/10.1063/1.4997574.

[24] J. Patera and P. Winternitz. Subalgebras of real three- and four-dimensional Lie algebras. Math. Phys., 88:1449-1455, 1977. https://doi.org/10.1063/1.523441.

[25] Y . Pomeau and P. Manneville. Wave length selection in cellular flows. Phys. Lett., 75(A):296-298, 1980. https://doi.org/10.1016/0375-9601(80)90568-X.

[26] W. Sarlet. A comment on 'Conservation laws of higher order nonlinear PDEs and the variational conservation laws in the class with mixed derivatives'. J. Phys. A Math. Theor., 43(45):458001, 2010. https://doi.org/10.1088/1751$8113 / 43 / 45 / 458001$.

[27] G.I. Sivashinsky. Instabilities, pattern-formation, and turbulence in flames. Ann. Rev. Fluid Mech., 15:179-199, 1983. https://doi.org/10.1146/annurev.fl.15.010183.001143.

[28] J. Swift and P. Hohenberg. Hydrodynamic fluctuations at the convective instability. Phys. Rev. A, 15:319-328, 1977. https://doi.org/10.1103/PhysRevA.15.319. 